\title{
A Multidimensional Cross-Sectional Analysis of Coronavirus Disease 2019 Seroprevalence Among a Police Officer Cohort: The PoliCOV-19 Study
}

\author{
Parham Sendi, ${ }^{1, \odot}$ Rossella Baldan, ${ }^{1, \odot}$ Marc Thierstein, ${ }^{2}$ Nadja Widmer, ${ }^{3}$ Peter Gowland, ${ }^{3, \odot}$ Brigitta Gahl, ${ }^{4, \odot}$ Annina Elisabeth Büchi, ${ }^{5, \odot}$ \\ Dominik Güntensperger, ${ }^{4, \odot}$ Manon Wider, ${ }^{1, \oplus}$ Manuel Raphael Blum, ${ }^{6,1, \odot}$ Caroline Tinguely, ${ }^{3, \odot}$ Cédric Maillat, ${ }^{8}$ Elitza S. Theel, ${ }^{9, \oplus}$ Elie Berbari, ${ }^{10, \odot}$ \\ Ronald Dijkman, ${ }^{1, \odot}$ and Christoph Niederhauser ${ }^{1,3, \odot}$
}

${ }^{1}$ Institute for Infectious Diseases, University of Bern, Bern, Switzerland, ${ }^{2}$ Division Operations, Cantonal Police Bern, Bern, Switzerland, ${ }^{3}$ Interregional Blood Transfusion, Swiss Red Cross, Bern, Switzerland, ${ }^{4}$ Clinical Trials Unit, CTU Bern, University of Bern, Bern, Switzerland, ${ }^{5}$ Department of Emergency Medicine, Inselspital, Bern University Hospital, University of Bern, Bern, Switzerland, ${ }^{6}$ Department of General Internal Medicine, Inselspital, Bern University Hospital, University of Bern, Bern, Switzerland, ${ }^{7}$ Institute of Primary Health Care (BIHAM), University of Bern, Bern, Switzerland, ${ }^{8}$ Hôpital du Jura bernois SA, Saint-Imier, Switzerland, ${ }^{9}$ Division of Clinical Microbiology, Mayo Clinic, Rochester, Minnesota, USA, and ${ }^{10}$ Division of Infectious Diseases, Mayo Clinic, Rochester, Minnesota, USA

Background. Protests and police fieldwork provide a high-exposure environment for severe acute respiratory syndrome coronavirus 2 (SARS-CoV-2) infections. In this cross-sectional analysis, we investigated the seroprevalence among a police cohort, and sociodemographic, work, and health-related factors associated with seropositivity.

Methods. Study participants were invited for serological testing of SARS-CoV-2 and to complete online questionnaires. Serum neutralization titers toward the wild-type SARS-CoV-2 spike protein (expressing D614G) and the Alpha and Beta variants were measured in seropositive study participants.

Results. A total of 978 police personnel representing 35\% of the entire staff participated from February to March 2021. The seroprevalence was $12.9 \%$. It varied by geographic region, ranged from $9 \%$ to $13.5 \%$ in 3 regions, including the city; and was $22 \%$ in Bernese Seeland/Jura with higher odds for seropositivity (odds ratio [OR], 2.38 [95\% confidence interval $\{\mathrm{CI}\}, 1.28-4.44], P=.006$ ). Job roles with mainly office activity were associated with a lower risk of seropositivity (OR, 0.33 [95\% CI, .14-.77], $P=.010$ ). Selfreported compliance with mask wearing during working hours was $100 \%$; $45 \%$ of seropositive vs $5 \%$ of seronegative participants $(P<.001)$ reported having had contact with a proven coronavirus disease 2019 (COVID-19) case living in the same household prior to serological testing. The level of serum antibody titers correlated with neutralization capacity. Antibodies derived from natural SARS-CoV-2 infection effectively neutralized the SARS-CoV-2 spike protein, but were less effective against the Alpha and Beta variants.

Conclusions. The seroprevalence of anti-SARS-CoV-2 antibodies of police officers was comparable to that reported in the general population, suggesting that the personal protective equipment of the police is effective, and that household contacts are the leading transmission venues. The level of serum antibody titers, in particular that of anti-spike antibodies, correlated well with neutralization capacity. Low antibody titers acquired from natural infection were not effective against variants.

Clinical Trials Registration. NCT04643444.

Keywords. anti-SARS-CoV-2 antibodies; coronavirus disease 2019; seroprevalence; police officers.

Serological surveys that detect antibodies against severe acute respiratory syndrome coronavirus 2 (SARS-CoV-2) antigens provide information on the prevalence in groups that might be more exposed to the virus or have had higher rates of infection

Received 20 September 2021; editorial decision 9 October 2021; accepted 13 October 2021; published online 16 October 2021.

Correspondence: Parham Sendi, MD, Institute for Infectious Diseases, University of Bern, Friedbühlstrasse 51,3010, Bern, Switzerland (parham.sendi@ifik.unibe.ch).

Open Forum Infectious Diseases ${ }^{\circledR} 2021$

(C) The Author(s) 2021. Published by Oxford University Press on behalf of Infectious Diseases Society of America. This is an Open Access article distributed under the terms of the Creative Commons Attribution-NonCommercial-NoDerivs licence (https://creativecommons.org/ licenses/by-nc-nd/4.0/), which permits non-commercial reproduction and distribution of the work, in any medium, provided the original work is not altered or transformed in any way, and that the work is properly cited. For commercial re-use, please contact journals.permissions@oup.com https://doi.org/10.1093/ofid/ofab524
[1]. They help researchers to quantify the protective effect of mitigation efforts. Although the majority of workers exposed to proven SARS-CoV-2 are employed in healthcare sectors, other occupations have been associated with an increased risk for coronavirus disease 2019 (COVID-19). Police officers are one such commonly exposed group. In contrast to healthcare workers, this special population has contact with a frequently changing and unpredictable population [2]. Physical distancing is often not possible. Unlike healthcare workers, police officers have no information pertaining to potential infectious diseases of the involved parties. Previous studies have shown that droplet and aerosol emission of contagious organisms occurs during speech [3-5] and increases with voice loudness [6]. These data underscore the risk of exposure to SARS-CoV-2 that may occur 
in fieldwork. This notion is even more important considering that the COVID-19 pandemic has ignited social unrest, including domestic violence and a surge in COVID-19 negations and anti-masking and anti-vaccine protests worldwide [7-12]. It is reasonable to hypothesize that police officers, in particular those working in the field, are a high-exposure population.

To assess the risk for COVID-19 in this group, we are studying a cohort consisting of individuals employed by the Cantonal Police Bern in Switzerland [13]. The aim of this cross-sectional analysis was to determine the seroprevalence of SARSCoV-2 antibodies in employees of the cantonal police and to investigate individual and work-related factors associated with seropositivity. We also measured antibody titers of naturally acquired SARS-CoV-2 infection and correlated the results with the neutralizing capacity of the antibodies towards the "wildtype" SARS-CoV-2 spike (S) protein (expressing D614G) and the Alpha and Beta variants.

\section{MATERIALS AND METHODS}

\section{SARS-CoV-2 Exposure}

\section{The First Wave.}

The first case of COVID-19 was confirmed in Switzerland on 25 February 2020. On 16 March 2020, schools and most businesses were closed nationwide. On 20 March 2020, all gatherings of $>5$ people in public spaces were banned. The measures were gradually removed between late April and June 2020. On 6 July 2020, the Federal Council ordered mandatory face masks on public transport for all individuals 12 years of age or older.

\section{The Second Wave.}

New measures were imposed in October 2020 as cases surged again.

\section{Mask Wearing.}

Wearing face masks for employees of the Cantonal Police Bern was recommended on 28 August 2020, and made mandatory during working hours on 13 October 2020. The types of mask provided by the police to their employees included surgical masks (type IIR) and police cloth masks certified by a material sciences and technology institute [14].

\section{Viral Strains.}

The viral strain consisting of the mutation D614G in the S protein (Nextstrain clade 20A and its descendants) was the dominating circulating variant in 2020 in Switzerland [15]. The Alpha variant (B.1.1.7) became the common variant in Switzerland starting in mid-February 2021 [16].

\section{Study Population}

The police force of the canton of Bern employs $>2800$ individuals, located across 58 police stations in cities and rural areas. The cantonal police have 4 regions of activity in bilingual (German and
French) areas, including the capital of the country (Bern city). The departments are subcategorized as regional, criminal, prevention and environment, and others. Rules and regulations on hygiene precautions and mask wearing were identical for all districts.

\section{Recruitment Period, Questionnaires, and Blood Sampling}

Study enrollment opened on 21 December 2020. Study participants who fulfilled the inclusion criteria (employees of the Cantonal Police Bern, aged 18-65 years) were asked to fill out 2 online questionnaires prior to being given appointments for blood sampling from 9 February to 9 March 2021. For every study participant, we collected coded, self-reported data on age and sex, education, job role within the police department, percentage employment, geographic region for work and living areas, and underlying health conditions. In addition, self-reported data on personal protective equipment use and hygiene precautions, symptoms consistent with COVID-19, contact with presumed or confirmed cases, quarantine, and nasopharyngeal test results were obtained. The vast majority of nasopharyngeal swab tests included polymerase chain reaction technology, because rapid antigen tests became available through health professionals in Switzerland in November 2020 and for use by the general population in April 2021 (ie, after recruiting and blood sampling). Answers to questions were provided prior to reporting the antibody test results.

After testing for the presence of antibodies, we contacted seropositive individuals again for an additional questionnaire on their subjective views about where the transmission possibly occurred.

\section{Antibody Tests}

SARS-CoV-2 antibodies to the nucleocapsid protein (NCP) and spike (S) protein were measured by using 2 commercially available immunoassays: anti-SARS-CoV-2 and anti-SARSCoV-2 S (Roche Diagnostics, Rotkreuz, Switzerland). Both immunoassays detect antibodies independent of isotype, detecting predominantly immunoglobulin $\mathrm{G}$ (IgG) antibodies, as well as immunoglobulin $\mathrm{A}$ and immunoglobulin $\mathrm{M}$ antibodies. The anti-SARS-CoV-2 assay is based on antibody detection to a recombinant SARS-CoV-2 NCP, whereas the anti-SARS-CoV-2 S uses a recombinant receptor binding domain antigen, which is found on subunit 1 of the spike protein. The NCP immunoassay reports a cutoff index (COI; signal of sample/cutoff) in which values $\geq 1.00$ are considered positive, whereas the $S$ immunoassay is a semi-quantitative method and reports results in absorbance units per milliliter $(\mathrm{U} / \mathrm{mL})$, for which values $\geq 0.8 \mathrm{AU} / \mathrm{mL}$ are considered positive. The tests were performed on the Roche Cobas 8000 e801 analyzer according to the manufacturer's instructions (Roche Diagnostics, Rotkreuz, Switzerland).

\section{Test Strategy for Analysis}

The cross-sectional baseline investigation was performed in a nonvaccinated population, because samples were obtained prior to initiating a vaccine program for the employees of the 
police. First, all study participants were tested for the presence of anti-NCP antibodies [17]. The rationale for this testing strategy included the high specificity and sensitivity of the anti-NCP electrochemiluminescence immunoassay (ECLIA) test evaluated in our laboratory $[18,19]$. Second, all anti-NCPseropositive samples were then also tested for anti-S antibodies. The rationale for testing anti-S antibodies in NCP-seropositive individuals relied on the intended neutralization assays and correlation statistics between anti-NCP and anti-S antibody titers. Among seronegative samples, only those from individuals who reported having had a positive test result from a nasopharyngeal swab in the online questionnaire were tested for anti-S antibodies (Supplementary Figure A).

\section{Serum Neutralization Assays}

The assays were performed as previously described [20]. In brief, 20000 Vero E6 cells were seeded in a 96-well plate format. The following day, heat-inactivated sera were 2 -fold serial diluted and mixed with 200 plaque-forming units of the indicated isogenic SARS-CoV-2 virus, which were generated, rescued, and propagated [20]. After 1 hour of preincubation at room temperature, the mixture was added to Vero E6 cells and incubated at $37^{\circ} \mathrm{C}$. After 4 days, cells were fixed with $4 \%$ formalin and stained with crystal violet to analyze the reciprocal dilution at which SARS-CoV-2 was neutralized. We used isogenic SARS-CoV-2 viruses harboring either the D614G spike, the full-length B.1.1.7 spike (Alpha variant), or the full-length B.1.351 spike (Beta variant).

\section{Statistical Analysis}

Seropositive individuals included those with a positive result and seronegative individuals those with a negative result in the anti-NCP antibody assay. The sample size calculation conducted prior to the study yielded a $95 \%$ confidence interval (CI) width of $4 \%$, assuming a true seroprevalence of $12 \%-13 \%$ and inclusion of 1000 study participants [13]. To describe characteristics of the study cohort, we used mean \pm standard deviation or median with interquartile range for summarizing continuous variables, as appropriate. Comparisons were made by using the Student $t$ test or Mann-Whitney test, respectively. Categorical data were shown as numbers with percentages and compared by using Fisher exact test for binary variables or the $\chi^{2}$ test for $>2$ categories, unless indicated as having been tested for a nonparametric trend, following the approach of Cuzick [21]. To investigate whether the outcome (seropositivity) was associated with a set of exposures, we used logistic regression on the entire cohort and on the subcohort of exposed subjects. We addressed the question of how well specific symptoms separate seropositive participants from those who tested negative by using random forests with 500 iterations. We calculated the odds ratio (OR) with the CI, sensitivity, specificity, and C statistic (area under the receiver operating characteristic curve) of the symptom with the highest importance. In seropositive patients, we visualized the correlation between antibody concentration (anti$\mathrm{NCP}$ and anti-S) with maximal dilution of the neutralization tests and calculated Spearman correlation coefficients. We also investigated whether anti-NCP and anti-S antibody titers were equally associated with neutralization serum titers of the 3 different virus variants by using multilevel mixed-effects ordered logistic regression (ie, the level of serum neutralization titers as ordered categories). We calculated separate analyses, including both anti-NCP and anti-S antibodies as covariates into the model as continuous variables or dichotomized by the median, respectively. All analyses were performed with Stata 16 software (StataCorp, College Station, Texas).

\section{Patient Consent Statement}

All participants gave written informed consent prior to enrollment in the PoliCOV-19 study. The design of the work was approved by the Cantonal Research Ethics Commission of Bern, Switzerland (ID-2020-02650).

\section{RESULTS}

A total of 989 individuals were enrolled in the study until 9 March 2021. Five individuals withdrew consent, and 6 missed the appointment for blood sampling. Hence, 978 employees of the Cantonal Police Bern were included in the final analysis, reflecting $35 \%$ of the entire staff. The proportion was representative in view of geographic distribution and job roles (data not shown). At the time of blood sampling, only 2 individuals were vaccinated with a messenger RNA vaccine (Supplementary Figure A). Both of them were anti-NCP seronegative.

\section{Seroprevalence}

A total of $852(87.1 \%)$ individuals were seronegative and $126(12.9 \%)$ were seropositive at their anti-NCP antibody assay (Table 1). Except for 2 individuals (1.6\%), all antiNCP-seropositive samples were also positive for anti-S IgG (Supplementary Figure A).

Seroprevalence varied by geographic region within the canton; it ranged from $9 \%$ to $13.5 \%$ in 3 regions, including the city, and was $22 \%$ in Bernese Seeland and Bernese Jura (Figure 1 and Table 1).

Individuals who worked in the Bernese Seeland and Bernese Jura regions had significantly higher odds of having anti-SARSCoV-2 antibodies than did those working in other regions (OR, 2.38 [95\% CI, 1.28-4.44]; Supplementary Table A).

High public exposure (eg, regional police with a high proportion of fieldwork activity) was associated with seropositivity (Table 1). Conversely, roles with mainly office activity (ie, interdepartmental) were associated with a lower risk of seropositivity (OR, 0.33 [95\% CI, .14-.77], $P=.010$; Supplementary Table B).

In 85 of $126(67.5 \%)$ seropositive individuals, the time interval between COVID-19 and blood sampling was 3 months 
Table 1. Demographic Characteristics and Anti-Nucleocapsid Protein Immunoglobulin G Status for 978 Employees of the Bern Cantonal Police ${ }^{\mathrm{a}, \mathrm{b}}$

\begin{tabular}{|c|c|c|c|c|c|}
\hline Characteristic & No. of Responses & Study Participants ( $N=978$ ) & Seropositive $(n=126)$ & Seronegative $(n=852)$ & $P$ Value \\
\hline Age, $y$, mean (SD) & 880 & $40(8.9)$ & $39(9.0)$ & $41(8.8)$ & .12 \\
\hline Sex & 973 & & & & .67 \\
\hline Female & & $270(28)$ & $37(29)$ & $233(27)$ & \\
\hline Male & & $703(72)$ & $89(71)$ & $614(72)$ & \\
\hline \multicolumn{6}{|l|}{ Comorbidity } \\
\hline $\mathrm{BMI}, \mathrm{kg} / \mathrm{m}^{2}$, mean (SD) & 975 & $26(3.5)$ & $26(3.3)$ & $26(3.5)$ & .46 \\
\hline Diabetes mellitus & 972 & $13(1.3)$ & $3(2.4)$ & $10(1.2)$ & .23 \\
\hline Arterial hypertension & 973 & $78(8.0)$ & $10(7.9)$ & $68(8.0)$ & 1.00 \\
\hline Cardiovascular disease & 971 & $17(1.7)$ & $3(2.4)$ & $14(1.6)$ & .47 \\
\hline Lung disease & 973 & $27(2.8)$ & $2(1.6)$ & $25(2.9)$ & .56 \\
\hline Immunosuppression & 971 & $12(1.2)$ & $2(1.6)$ & $10(1.2)$ & .66 \\
\hline Other disease & 973 & $101(10)$ & $10(7.9)$ & $91(11)$ & .43 \\
\hline No comorbidity & 978 & $761(78)$ & $100(79)$ & $661(78)$ & .73 \\
\hline Education & 974 & & & & .16 \\
\hline Police academy & & $795(81)$ & $114(90)$ & $681(80)$ & \\
\hline Security assistant school & & $53(5.4)$ & $4(3.2)$ & $49(5.8)$ & \\
\hline University degree & & $63(6.4)$ & $3(2.4)$ & $60(7.0)$ & \\
\hline Merchant & & $32(3.3)$ & $2(1.6)$ & $30(3.5)$ & \\
\hline Craftsman & & $18(1.8)$ & $2(1.6)$ & $16(1.9)$ & \\
\hline Other & & $13(1.3)$ & $1(0.79)$ & $12(1.4)$ & \\
\hline No. of years working for the police, median (IQR) & 915 & $11(7.0-19)$ & $11(7.0-20)$ & $11(6.5-19)$ & .75 \\
\hline Working region within the canton & 963 & & & & .006 \\
\hline Seeland, Bernese Jura & & $170(17)$ & $37(29)$ & $133(16)$ & \\
\hline Mittelland, Emmental, Oberaargau & & $193(20)$ & $22(17)$ & $171(20)$ & \\
\hline Bernese Oberland & & $132(13)$ & $14(11)$ & $118(14)$ & \\
\hline Region Bern & & $229(23)$ & $31(25)$ & $198(23)$ & \\
\hline Bern City & & $239(24)$ & $22(17)$ & $217(25)$ & \\
\hline Department & 973 & & & & .017 \\
\hline Regional police & & $649(66)$ & $90(71)$ & $559(66)$ & \\
\hline Criminal police & & $118(12)$ & $20(16)$ & $98(12)$ & \\
\hline Traffic, environment, prevention & & $72(7.4)$ & $10(7.9)$ & $62(7.3)$ & \\
\hline Interdepartmental & & $120(12)$ & $6(4.8)$ & $114(13)$ & \\
\hline Other & & $14(1.4)$ & $0(0.00)$ & $14(1.6)$ & \\
\hline Main activity & 939 & & & & .038 \\
\hline Fieldwork & & $559(57)$ & $84(67)$ & $475(56)$ & \\
\hline Office work & & $380(39)$ & $39(31)$ & $341(40)$ & \\
\hline$\%$ of working hours in the field, median (IQR) & 952 & $50(20-70)$ & $60(30-70)$ & $50(20-70)$ & .032 \\
\hline$\%$ of working hours in the office, median (IQR) & 976 & $50(30-80)$ & $40(30-70)$ & $50(30-80)$ & .012 \\
\hline
\end{tabular}

Data are presented as No. (\%) unless otherwise indicated. Values in bold are statistically significant $(P<.05)$.

Abbreviations: BMI, body mass index; IQR, interquartile range; SD, standard deviation.

${ }^{a}$ All except 2 individuals with anti-nucleocapsid protein (NCP) antibodies also displayed anti-spike antibodies (Supplementary Figure A).

${ }^{\mathrm{b}}$ Two vaccinated individuals (both anti-NCP antibody negative) were included in this analysis.

or longer. Twenty-four (19\%) study participants did not know when their infection occurred. There was no difference in anti-NCP and anti-S antibody titer results when we compared ECLIA values with the time intervals of 1 month, 2 months, 3 months, and longer (Supplementary Figure B).

Ninety-nine individuals reported having had a prior positive nasopharyngeal swab test result; 94 of them (95\%) were seropositive for anti-NCP and 96 (97\%) for anti-S antibodies. Only 3 (3\%) of the positive tested individuals showed no seroconversion (Supplementary Table C). Three hundred thirty-six individuals reported never having had COVID-19 symptoms and that they were not tested; 329 (98\%) of them were seronegative for anti-NCP antibodies.

\section{Symptoms}

Symptoms consistent with COVID-19 were significantly more frequent in the seropositive than in the seronegative group (Table 2). Among reported symptoms, "new loss of smell or taste" was the best discriminator (Supplementary Figure C). This symptom was associated with an OR of 52.4 (95\% CI, $30.9-89.0 ; P<.001)$ for seropositivity, sensitivity of $64 \%(95 \%$ CI, $55 \%-72 \%$ ), specificity of $97 \%$ (95\% CI, $95 \%-98 \%)$, and an 


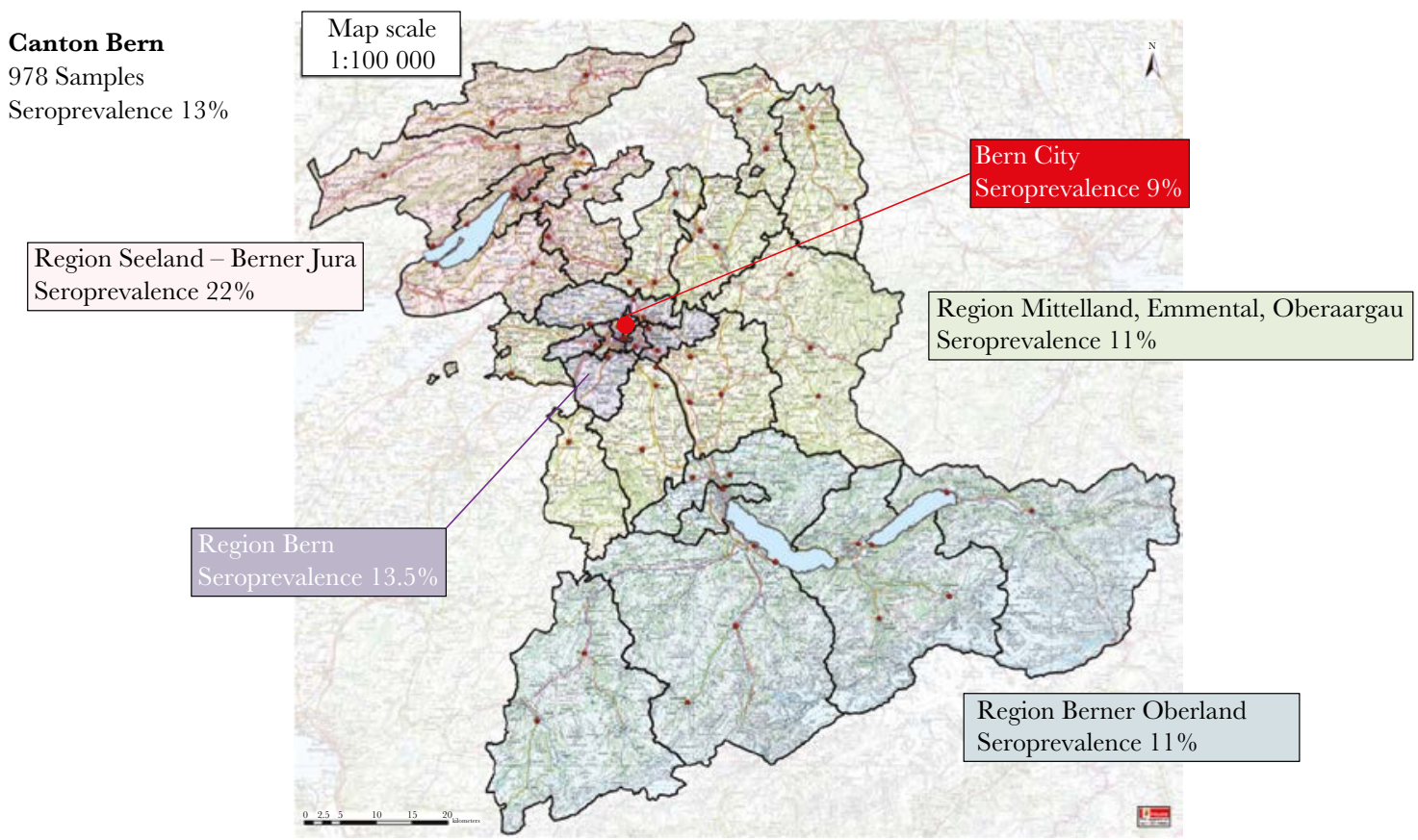

Figure 1. Map of the canton of Bern in Switzerland. The different colors indicate the corresponding geographic regions. The overall seroprevalence was $12.9 \%$ (ie, 126 of 978 samples displayed anti-nucleocapsid protein antibodies). Responses of 963 study participants were available; 15 seronegative individuals did not provide their geographic working district.

area under the receiver operating characteristic curve of $80 \%$ (95\% CI, 76\%-85\%).

Personal Protective Equipment Use, Absenteeism, Quarantine, and Transmission Venues

Reported compliance with wearing masks during working hours was $100 \%$, irrespective of the presence of anti-SARSCoV-2 antibodies (Supplementary Table D).

Two hundred forty-four individuals (25\%) missed work (including home office) or were absent from the police academy because of suspected or confirmed COVID-19 or because of quarantine. Two hundred twenty-four (23\%) individuals were placed in quarantine because of exposure to a person with proven or suspected COVID-19, and 65 (29\%) were subsequently seropositive. These 65 employees represented 52\% of all seropositive individuals in the study, while the other 159 employees who were placed in quarantine (and who remained seronegative) reflected $19 \%$ of all seronegative study participants $(P<.001)$.

The investigation on possible transmission venues specifically differentiated between contacts during working hours

Table 2. Clinical Symptoms Reported by Study Participants During the Coronavirus Disease 2019 Pandemic

\begin{tabular}{|c|c|c|c|c|c|}
\hline Symptoms & No. of Responses & Study Participants $(\mathrm{N}=978)^{\mathrm{a}}$ & Seropositive $(n=126)$ & Seronegative $(n=852)$ & PValue \\
\hline Fever & 954 & $130(13)$ & $41(33)$ & $89(11)$ & $<.001$ \\
\hline Chills & 949 & $137(14)$ & 41 (33) & $96(11)$ & $<.001$ \\
\hline Fatigue & 959 & $547(56)$ & $102(81)$ & $445(53)$ & $<.001$ \\
\hline Muscle or body aches & 947 & $142(15)$ & $47(37)$ & $95(11)$ & $<.001$ \\
\hline Sore throat & 963 & $406(42)$ & $56(44)$ & $350(41)$ & .56 \\
\hline Congestion or runny nose & 959 & $479(49)$ & $73(58)$ & $406(48)$ & .045 \\
\hline New loss of taste or smell & 948 & $107(11)$ & $80(63)$ & $27(3.2)$ & $<.001$ \\
\hline Shortness of breath or difficulty breathing & 948 & $135(14)$ & $42(33)$ & $93(11)$ & $<.001$ \\
\hline Chest pain & 947 & $129(13)$ & $33(26)$ & $96(11)$ & $<.001$ \\
\hline Cough or other respiratory symptoms & 937 & $84(8.6)$ & $20(16)$ & $64(7.6)$ & .004 \\
\hline Headache & 955 & $263(27)$ & $75(60)$ & $188(22)$ & $<.001$ \\
\hline Nausea or vomiting & 945 & $65(6.7)$ & $13(10)$ & $52(6.1)$ & .09 \\
\hline Abdominal pain & 941 & $71(7.3)$ & $8(6.3)$ & $63(7.4)$ & .85 \\
\hline Diarrhea & 945 & $133(14)$ & $26(21)$ & $107(13)$ & .018 \\
\hline
\end{tabular}

Data are presented as No. (\%) unless otherwise indicated. Values in bold are statistically significant $(P<.05)$

${ }^{a}$ Two vaccinated individuals (both anti-nucleocapsid protein antibody negative) were included in this analysis. 
Table 3. Comparison of Seropositive (ie, Seroconversion) and Seronegative (ie, No Seroconversion) Individuals After Contact With a Proven or Presumed Coronavirus Disease 2019 Case

\begin{tabular}{|c|c|c|c|c|c|}
\hline Reasons for Being in Quarantine & $\begin{array}{l}\text { No. of Re- } \\
\text { sponses }\end{array}$ & $\begin{array}{c}\text { Study Partici- } \\
\text { pants }(\mathrm{N}=978)^{\mathrm{a}}\end{array}$ & $\begin{array}{l}\text { Seropositive } \\
(\mathrm{n}=126)\end{array}$ & $\begin{array}{l}\text { Seronegative } \\
\quad(\mathrm{n}=852)\end{array}$ & $\begin{array}{c}P \\
\text { Value }\end{array}$ \\
\hline $\begin{array}{l}\text { Contact with a person living in the same household who } \\
\text { had COVID-19 (proven with a test) }\end{array}$ & 978 & $102(10)$ & $57(45)$ & $45(5.3)$ & $<.001$ \\
\hline $\begin{array}{l}\text { Contact with a person living in the same household who } \\
\text { had presumable COVID-19 (not proven with a test) }\end{array}$ & 977 & $105(11)$ & $20(16)$ & $85(10)$ & .06 \\
\hline $\begin{array}{l}\text { Contact with a person at work who had COVID-19 } \\
\text { (proven with a test) }\end{array}$ & 977 & 364 (37) & 43 (34) & 321 (38) & .49 \\
\hline $\begin{array}{l}\text { Contact with a person at work who had presumable } \\
\text { COVID-19 (not proven with a test) }\end{array}$ & 976 & $192(20)$ & $22(17)$ & $170(20)$ & .55 \\
\hline
\end{tabular}

Data are presented as No. (\%) unless otherwise indicated. Values in bold are statistically significant $(P<.05)$.

Abbreviation: COVID-19, coronavirus disease 2019 .

${ }^{\mathrm{a}}$ Two vaccinated individuals (both anti-nucleocapsid protein antibody negative) were included in this analysis.

and household, and between "presumed" and "proven" (ie, confirmed with a nasopharyngeal swab test) COVID-19 contact. Fifty-seven of 126 (45\%) seropositive individuals reported having had contact with a proven COVID-19 case living in the same household. Conversely, $5 \%$ of all seronegative individuals reported the same type of exposure. This proportion difference was statistically significant $(P<.001)$. This was not the case when reported contacts during working hours or contacts with presumed (but not proven) cases were analyzed (Table 3 ).

Subjective Assessment of Study Participants on the Source of Transmission Among seropositive individuals, 75 (60\%) felt "certain" or "likely certain" about the source of transmission (Supplementary Table E). Sixty-one (48\%) reported that the contact had occurred during a private activity or within the same household.

\section{Neutralization Capacity and Correlation With ECLIA Results}

The required dilution titers for neutralization showed a considerable distribution among the study participants (Figure 2). The median dilution titer was significantly higher in assays that used D614G than in those that used B.1.1.7 $(P<.001)$ and B.1.351 $(P<.001)$. Similarly, the median dilution titer was significantly higher in assays that used B.1.1.7 than in those that used B.1.351 $(P<.001)$. Antibody titers of anti-NCP and anti-S antibodies correlated with the dilution titers showing the highest coefficient with dilution of D614G and the lowest coefficient with dilution of B.1.351 (Beta), with $P<.001$ in all pairs (Supplementary Table F). Antibodies from seropositive individuals demonstrated neutralization activity against D614G up to a dilution of 1:320 (Supplementary Figure D). The dilutions were lower for B.1.1.7 (1:40 for anti-S antibodies and 1:80 for anti-NCP antibodies) and for B.1.351 variants (1:20) (Supplementary Figures E and F),

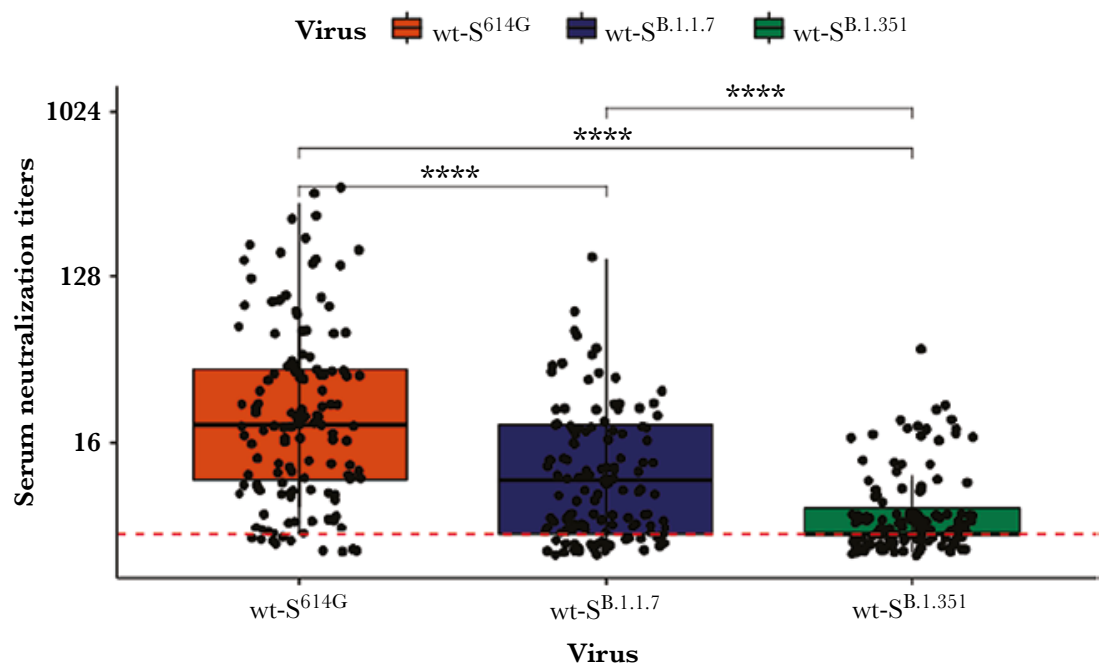

Figure 2. Results of neutralization assays performed with serum of study participants $(n=126)$ and isogenic severe acute respiratory syndrome coronavirus 2 viruses harboring either the D614G spike, the full-length B.1.1.7 spike (Alpha variant), or the full-length B.1.351 spike (Beta variant). Each dot represents the results of a study participant. Each sample was tested against all 3 strains. The red dashed line reflects the limit of detection. The numbers on the $y$-axis indicate the highest dilution of serum demonstrating neutralization activity. The box plots display the distribution of data between the first quartile, median, and third quartile. A Wilcoxon rank-sum and signed-rank test was performed to compare the groups. $* * * * P .0001$. Abbreviation: wt-S, wild-type spike. 


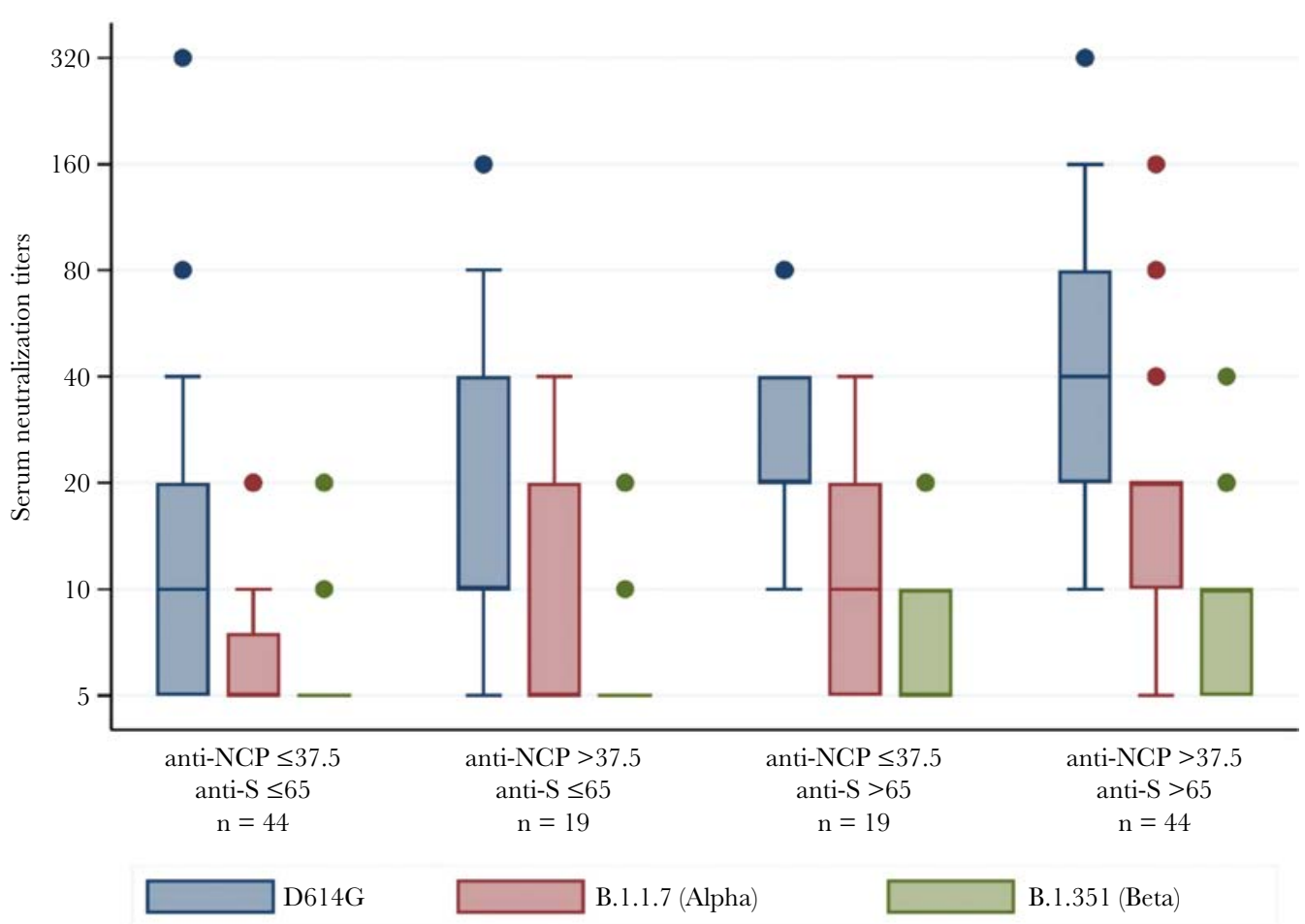

Figure 3. Results of neutralization assays categorized according to selected cutoff electrochemiluminescence immunoassay test values of anti-nucleocapsid protein (NCP) antibodies (cutoff index $\leq 37.5$ and >37.5) and anti-spike (S) antibodies ( $\leq 65 \mathrm{U} / \mathrm{mL}$ and $>65 \mathrm{U} / \mathrm{mL}$ ). The combination of these values result in 4 groups. The box plots display the distribution of data between the first quartile, median, and third quartile of serum neutralization titers of each group. The numbers on the $y$-axis indicate the highest serum dilution demonstrating neutralization activity.

indicating a poorer neutralization capacity toward virus variants in comparison to the main circulating virus expressing D614G.

We then searched for cutoff values of anti-NCP antibodies and anti-S antibodies in ECLIA results that demonstrated neutralization in the assay. We explored antibody titers as both continuous and binary variables in separate statistical models to mathematically predict the level of neutralization. For the latter, we used the median values of all results (ie, $>37.5 \mathrm{COI}$ for anti-NCP antibodies and $>65 \mathrm{U} / \mathrm{mL}$ for anti-S antibodies) and hence, assembled 4 combination categories (Figure 3). Similar to the overall results of neutralization assays, the neutralization capacity of serum against Alpha and Beta variants was poorer than it was against D614G, even with serum demonstrating both $>37.5 \mathrm{U} / \mathrm{mL}$ COI anti-NCP antibodies and $>65 \mathrm{U} / \mathrm{mL}$ anti-S antibodies. In the model with both continuous and ordered categories, ORs of anti-S antibodies for the level of neutralization were higher than those of anti-NCP antibodies. While the antibody titers above the cutoff level of $>37.5 \mathrm{U} / \mathrm{mL}$ were associated with about a 3-fold increase in level of neutralization, anti-S antibody titers $>65 \mathrm{U} / \mathrm{mL}$ were associated with about a 6-fold increase (Supplementary Table G).

\section{DISCUSSION}

This cross-sectional population serological survey among the police cohort demonstrated a prevaccinated
anti-SARS-CoV-2 antibody seroprevalence of 13\%. Few studies have investigated seroprevalence in police officers, which include those performed in New York City [22] and in Mazowieckie Province, Poland [23], as well as 2 further studies with low sample sizes $[24,25]$. However, our study did not demonstrate a higher seroprevalence than observed in the general population of the canton of Bern (ie, 14\%), which was investigated in another study using a different serological test $[26,27]$. The results indicate that the use of personal protective equipment is effective in mitigating the risk of COVID-19. This is in line with the reported high compliance with mask wearing in our study population. However, within the police cohort, the odds for seropositivity were higher for fieldwork activity with high exposure to the general population than they were for office work activity with low exposure to the general population. The significant seroprevalence difference observed for contacts in the private environment and when comparing geographic districts is in line with the findings of others [22, 28, 29].

During the first wave of the pandemic in Switzerland, testing individuals with few or no symptoms was not recommended, and symptoms consistent with COVID-19 were presumed to be COVID-19 related. In the police cohort, nearly $50 \%$ of individuals experienced sore throat, congestion, or a runny nose, although only $5 \%-7 \%$ were seropositive, underscoring the 
importance of testing, considering that numerous other viruses can cause a variety of respiratory symptoms. In our analysis, new loss of taste or smell was associated with high specificity in predicting seropositivity. Following proven infection with SARS-CoV-2, most individuals in our cohort developed detectable serum antibodies toward both the NCP and the S protein. To assess ex-vivo humoral immunity within our cohort, we investigated the magnitude of neutralizing antibodies toward SARS-CoV-2 strains that did and did not circulate within the population prior to blood sampling.

Considering that the sampling occurred from 9 February to 9 March, the vast majority of our cohort was exposed to the virus strain harboring the D614G S protein [15]. Exposure to the Alpha and Beta variants was unlikely in our cohort [16], in particular when considering the reported time points of infection (Supplementary Figure B). In line with these observations, naturally acquired antibodies demonstrated good neutralization activity against D614G but performed suboptimally against the Alpha variant and poorly against the Beta variant. We observed a correlation between the ECLIA titers and the highest dilutions still demonstrating neutralization. Our results imply that the interaction between the spike protein and anti-S antibodies may play an important role in the neutralization tests. They point toward the importance of high anti-S antibody titers, and hence, the value of the vaccine achieving this goal. Although the variants show mutations mainly in the spike protein, variations in the capsule have gained less attention. In line with our results, the contribution to virus neutralization of anti-NCP antibodies is less known [30].

Our study has limitations. The study population may contain a response bias in comparison to the nonparticipants and nonresponders within the police staff. The investigation was performed in February 2021 and we used anti-NCP antibodies as the main marker of seropositivity. We cannot exclude that in certain individuals with COVID-19 in 2020 anti-NCP antibodies have waned below the detection level and that the true seroprevalence is underestimated. In our experience, the antibody titers remain at a detectable level for a prolonged period, and the proportion of agreement with reported nasopharyngeal swab test results was high. Therefore, and in consideration of a precalculated sample size and $95 \%$ CI width of $4 \%$, we are convinced that the seroprevalence proportion found in our analysis is a valid result. We categorized our analysis only in anti-NCPseropositive and seronegative individuals and did not correct for sensitivity and specificity. Two individuals with a history of a positive nasopharyngeal tests had positive anti-S without anti-NCP antibodies, and our test strategy did not include dual testing for all study participants (Supplementary Figure A). It is therefore possible that we included very few false-positive or false-negative serum samples in our analysis. Given the high sensitivity and specificity of the antibody tests $[19,31]$, we do not believe that excluding these few samples would have changed the overall results. The results from nasopharyngeal swab tests were obtained via online questionnaire, and the questionnaires on the subjective view of transmission routes may consist of a recall bias. Moreover, the directionality of transmission remains unknown, and identifying index and contact cases is subject to testing and reporting bias. The choice of median cutoff values of antibody titers in association with serum neutralization assays is arbitrary and does not reflect clinical circumstances. More sophisticated methods to find an optimal cutoff such as receiver operating characteristic curves would have required a fixed and already established cutoff for serum neutralization titer. We chose a conservative cutoff, supported by the sensitivity analysis with antibody titers as a continuous independent variable.

In conclusion, our COVID-19 cross-sectional survey among police officers demonstrated a seroprevalence of $13 \%$ in a prevaccinated cohort. This proportion is similar to that reported in the general population. The high compliance with mask wearing and the low proportion of seroconversion after contact with a presumed or proven COVID-19 case during working hours may imply that personal protective equipment is effective. The high proportion of seropositive individuals who have had contact with a proven COVID-19 case in the same household suggests that most known transmissions within our police cohort did not occur within working hours. The level of serum antibody titers, in particular that of anti-S antibodies, correlated well with the neutralization capacity. Antibodies derived from natural SARS-CoV-2 antibodies effectively neutralized viral strains that-from an epidemiological point of view-most likely caused the infection. However, at low titers (ie, below the median of the study population), antibodies were not effective against the Alpha and Beta variants. These findings support vaccine programs for both seropositive and seronegative individuals to maintain public protective services on a stably staffed level.

\section{Supplementary Data}

Supplementary materials are available at Open Forum Infectious Diseases online. Consisting of data provided by the authors to benefit the reader, the posted materials are not copyedited and are the sole responsibility of the authors, so questions or comments should be addressed to the corresponding author.

\section{Notes}

Acknowledgments. We thank Dr Volker Thiel (Institute of Virology and Immunology and Department of Infectious Diseases and Pathobiology, Vetsuisse Faculty, University of Bern, Bern, Switzerland) for providing the viral strains. We thank the numerous volunteers and the study team members from the affiliated and other institutions for their help in conducting the study, including Medbase Zweisimmen, Medicentre Moutier, and numerous others. We thank all the employees of the Cantonal Police Bern for participating. We are grateful to Annetta Redmann, Clinical Trials Unit, University of Bern, for her databank and data management. BioMedical Editor, St Albert, Alberta, Canada, provided English-language editing.

Disclaimer. The funding party had no influence in the study design, interpretation of results, or generation of the manuscript. The manufacturer of the electrochemiluminescence immunoassay tests (Roche) provided 
no funding for this study, as the test was commercially purchased by the investigators.

Financial support. The study was funded in part by the Cantonal Police of Bern, Bern, Switzerland. The Institute for Infectious Diseases of the University of Bern and the Interregional Blood Transfusion, Swiss Red Cross, Bern, Switzerland, supported the study by providing working hours of their employees specifically for this study and by providing material and consumables at cost or for free.

Potential conflicts of interest. All authors: No reported conflicts of interest.

All authors have submitted the ICMJE Form for Disclosure of Potential Conflicts of Interest. Conflicts that the editors consider relevant to the content of the manuscript have been disclosed.

\section{References}

1. Ong DSY, Fragkou PC, Schweitzer VA, et al; European Society of Clinical Microbiology and Infectious Diseases (ESCMID) Study Group for Respiratory Viruses (ESGREV). How to interpret and use COVID-19 serology and immunology tests. Clin Microbiol Infect 2021; 27:981-6.

2. Baker MG, Peckham TK, Seixas NS. Estimating the burden of United States workers exposed to infection or disease: a key factor in containing risk of COVID19 infection. PLoS One 2020; 15:e0232452.

3. Loudon RG, Roberts RM. Singing and the dissemination of tuberculosis. Am Rev Respir Dis 1968; 98:297-300.

4. Asadi S, Wexler AS, Cappa CD, et al. Effect of voicing and articulation manner on aerosol particle emission during human speech. PLoS One 2020; 15:e0227699.

5. Jones RM, Brosseau LM. Aerosol transmission of infectious disease. J Occup Environ Med 2015; 57:501-8.

6. Asadi S, Wexler AS, Cappa CD, et al. Aerosol emission and superemission during human speech increase with voice loudness. Sci Rep 2019; 9:2348.

7. Bradbury-Jones C, Isham L. The pandemic paradox: the consequences of COVID-19 on domestic violence. J Clin Nurs 2020; 29:2047-9.

8. Boserup B, McKenney M, Elkbuli A. Alarming trends in US domestic violence during the COVID-19 pandemic. Am J Emerg Med 2020; 38:2753-55.

9. Mazza M, Marano G, Lai C, et al. Danger in danger: interpersonal violence during COVID-19 quarantine. Psychiatry Res 2020; 289:113046

10. Roesch E, Amin A, Gupta J, García-Moreno C. Violence against women during covid-19 pandemic restrictions. BMJ 2020; 369:m1712.

11. Taylor L. Covid-19: Vaccine corruption allegations spark protests across Brazil. BMJ 2021; 374:n1724.

12. Fasehun LO. Reviewing COVID-19 modelling amidst recent United States protests. Ann Glob Health 2020; 86:76.

13. Sendi P. Police officer COVID-19 seroprevalence survey in the Canton of Bern, Switzerland. In: ClinicalTrials.gov (NCT04643444). https://clinicaltrials.gov/ct2/ show/NCT04643444. Accessed 17 October 2021.

14. Empa. ReMask: protective masks for Switzerland. 2021. https://www.empa.ch/ web/s604/remask. Accessed 17 October 2021.
15. Hodcroft EB, Zuber M, Nadeau S, et al. Spread of a SARS-CoV-2 variant through Europe in the summer of 2020. Nature 2021; 595:707-12

16. Federal Office of Public Health. COVID-19 Switzerland: information on the current situation. 2021. https://www.covid19.admin.ch/en/epidemiologic/virusvariants/d/overview. Accessed 17 October 2021.

17. Burbelo PD, Riedo FX, Morishima C, et al. Sensitivity in detection of antibodies to nucleocapsid and spike proteins of severe acute respiratory syndrome coronavirus 2 in patients with coronavirus disease 2019. J Infect Dis 2020; 222:206-13.

18. Riester E, Krieter B, Findeisen P, et al. Performance of an automated anti-SARSCoV-2 immunoassay in prepandemic cohorts. medRxiv [Preprint]. Posted online 7 August 2020. doi: 10.1101/2020.08.07.20169987.

19. Riester E, Majchrzak M, Mühlbacher A, et al. Multicentre performance evaluation of the Elecsys anti-SARS-CoV-2 immunoassay as an aid in determining previous exposure to SARS-CoV-2 [manuscript published online ahead of print 9 August 2021]. Infect Dis Ther 2021. doi: 10.1007/s40121-021-00504-9.

20. Ulrich L, Halwe NJ, Taddeo A, et al. Enhanced fitness of SARS-CoV-2 variant of concern B.1.1.7, but not B.1.351, in animal models. bioRxiv [Preprint]. Posted online 28 June 2021. doi: 10.1101/2021.06.28.450190.

21. Cuzick J. A Wilcoxon-type test for trend. Stat Med 1985; 4:87-90.

22. Sami S, Akinbami LJ, Petersen LR, et al. Prevalence of SARS-CoV-2 antibodies in first responders and public safety personnel, New York City, New York, USA, May-July 2020. Emerg Infect Dis 2021; 27:796-804.

23. Gujski M, Jankowski M, Pinkas J, et al. Prevalence of current and past SARSCoV-2 infections among police employees in Poland, June-July 2020. J Clin Med 2020; 9:3245.

24. Halatoko WA, Konu YR, Gbeasor-Komlanvi FA, et al. Prevalence of SARSCoV-2 among high-risk populations in Lomé (Togo) in 2020. PLoS One 2020; 15:e242124.

25. Chughtai OR, Batool H, Khan MD, Chughtai AS. Frequency of COVID-19 IgG antibodies among special Police Squad Lahore, Pakistan. J Coll Physicians Surg Pak 2020; 30:735-9.

26. Universität Bern. Only $14 \%$ of the Bernese population have antibodies against coronavirus. https://www.unibe.ch/news/media_news/media_relations_e/ media_releases/2021/media_releases_2021/only_14_of_the_bernese_population_have_antibodies_against_coronavirus/index_eng.html. Accessed 17 October 2021.

27. West EA, Anker D, Amati R, et al; Corona Immunitas Research Group. Corona Immunitas: study protocol of a nationwide program of SARS-CoV-2 seroprevalence and seroepidemiologic studies in Switzerland. Int J Public Health 2020; 65:1529-48.

28. Fung HF, Martinez L, Alarid-Escudero F, et al. The household secondary at tack rate of SARS-CoV-2: a rapid review. Clin Infect Dis 2021; 73(Suppl 2):S138-45

29. Madewell ZJ, Yang Y, Longini IM Jr, et al. Household transmission of SARS-CoV-2: a systematic review and meta-analysis. JAMA Netw Open 2020; 3:e2031756.

30. Brochot E, Demey B, Touzé A, et al. Anti-spike, anti-nucleocapsid and neutralizing antibodies in SARS-CoV-2 inpatients and asymptomatic individuals. Front Microbiol 2020; 11:584251.

31. Riester E, Findeisen P, Hegel JK, et al. Performance evaluation of the Roche Elecsys anti-SARS-CoV-2 S immunoassay. J Virol Methods 2021; 297:114271. 